\title{
Commentary: Ongoing advancements in the understanding of tricuspid valve dynamics and functional geometry
}

\author{
Daniel J. P. Burns, MD, MPhil
}

\author{
From the Department of Thoracic and Cardiovascular Surgery, Cleveland Clinic Foundation, Cleveland, Ohio. \\ Disclosures: Author has nothing to disclose with regard to commercial support. \\ Received for publication Sept 8, 2018; accepted for publication Sept 11, 2018; available ahead of print Nov 2, \\ 2018. \\ Address for reprints: Daniel J. P. Burns, MD, MPhil, Cleveland Clinic, 9500 Euclid Ave, J4-133, Cleveland, OH \\ 44195 (E-mail: burnsd@ccf.org). \\ J Thorac Cardiovasc Surg 2019;157:1463-4 \\ $0022-5223 / \$ 36.00$ \\ Copyright (C) 2018 by The American Association for Thoracic Surgery \\ https://doi.org/10.1016/j.jtcvs.2018.09.031
}

In developing prosthetics for valve repair, it is essential to understand the underlying dynamic functional nature of the valve in question, along with its essential geometric anatomic relationships. For the tricuspid valve, repair that uses a prosthetic ring or band has, through the years, become the criterion standard. Many prosthetic rings and bands exist to this end, although they vary in relative geometry and flexibility.

Recognizing these concerns, as they describe in their article in this issue of the Journal, Malinowski and colleagues ${ }^{1}$ have set out to define better the complex fine dynamic relationships that are present in the functioning human tricuspid valve. Previous geometric studies have used computed tomography, magnetic resonance, or echocardiography. Malinowski and colleagues ${ }^{1}$ note the limitations of these modalities, namely the requirement for multiplanar reconstruction or frame rate and resolution, respectively. To address this problem, Malinowski and colleagues ${ }^{1}$ have developed a human model that uses ex vivo perfusion and surgically implanted sonomicrometry crystals, a protocol previously used in animal models. ${ }^{2,3}$ Crystals implanted along the tricuspid annulus, right ventricular free wall, and right ventricular apex provide dynamic anatomic detail, while pressure transducers placed in the right atrium and ventricle provide hemodynamic data. Harvested hearts rejected for transplant, with no greater than mild right ventricular dysfunction or mild tricuspid regurgitation, are connected to an ex vivo perfusion system. Aortic inflow of donor blood provides perfusion, coronary sinus return volume loads the right ventricle in a closed right atrium, and pressure loading of the right ventricle is achieved by variable occlusion of pulmonary artery outflow. With this methodology, Malinowski and colleagues ${ }^{1}$ have demonstrated detailed results regarding the functional geometry and strain of the tricuspid annulus, as well as its dynamics during the cardiac cycle. They have also demonstrated

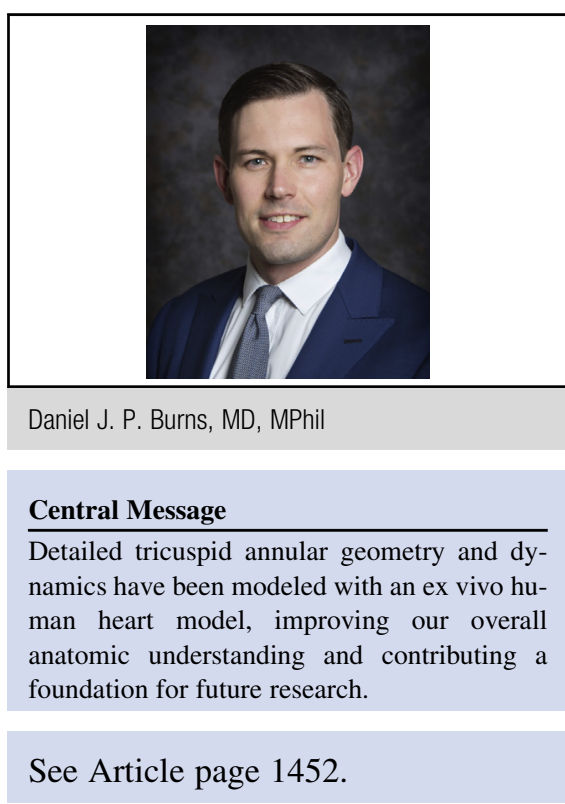

similar spatial relationships with contractile stress induced by calcium administration.

These results must be considered in the context of several important limitations, about which Malinowski and colleagues ${ }^{1}$ have been quite candid. This is an ex vivo human heart model, necessitated by the requirement for surgically implanted crystals and a wired connection. The hemodynamics therefore will differ from a true human heart, most notably because of the requirement for pacing and lack of left ventricular loading. Unloading may not affect tricuspid annular geometry meaningfully, as demonstrated in previous work by this group. ${ }^{4}$ With a less robust annulus relative to the mitral valve, pacing could potentially alter this geometry. This study also consisted of 11 donor hearts, with only 5 contributing to the statistical comparison before and after calcium administration, bringing up issues of sample size adequacy. Similarly, the small numbers included in a repeated measures analysis bring up issues of spurious results in statistical comparisons.

With a basic science study such as this, the reader is left asking, "So what?" In this case, this study elegantly demonstrates the dynamic geometry and function of the tricuspid annulus and helps to resolve conflicting functional anatomic descriptions. To take a translational approach, an improved understanding of the functional geometry and dynamics of the tricuspid annulus informs 
the future development of new tricuspid annular prostheses. Our current tools are good, but we should always be striving for better.

\section{References}

1. Malinowski M, Jazwiec T, Goehler M, Quay N, Bush J, Jovinge S, et al Sonomicrometry-derived 3-dimensional geometry of the human tricuspid annulus. J Thorac Cardiovasc Surg. 2019;157:1452-61.e1.
2. Malinowski M, Schubert H, Wodarek J, Ferguson H, Eberhart L, Langholz D, et al. Tricuspid annular geometry and strain after suture annuloplasty in acute ovine right heart failure. Ann Thorac Surg. 2018;106:1804-11.

3. Jazwiec T, Malinowski M, Proudfoot AG, Eberhart L, Langholz D, Schubert $\mathrm{H}$, et al. Tricuspid valvular dynamics and 3-dimensional geometry in awake and anesthetized sheep. J Thorac Cardiovasc Surg. 2018;156: 1503-11.

4. Malinowski M, Wilton P, Khaghani A, Brown M, Langholz D, Hooker V, et al. The effect of acute mechanical left ventricular unloading on ovine tricuspid annular size and geometry. Interact Cardiovasc Thorac Surg. 2016;23:391-6. 